\title{
Práticas interdisciplinares na promoção da saúde da pessoa idosa
}

\author{
Interdisciplinary practices in promoting the health of older adults \\ Prácticas interdisciplinarias en la promoción de la salud de las personas mayores
}

\author{
Milena Silva Costa ; Eliane de Sousa Leite ${ }^{\text {II }}$ Jamili Anbar Torquato ${ }^{\text {III }}$; Iluska Pinto Costa ${ }^{\text {IV }}$; \\ Ana Margareth Marques Fonseca Sarmento ${ }^{V}$; Maria Adelaide Silva Paredes Moreira ${ }^{V I}$
}

\begin{abstract}
RESUMO: O objetivo foi conhecer as práticas de promoção da saúde desenvolvidas pela equipe interdisciplinar com um grupo de idosos. Pesquisa quantiqualitativa, descritiva, realizada com 12 profissionais de saúde do grupo de idosos Amigos de Irmã Fernanda, de Cajazeiras - Paraíba, que responderam a um questionário em 2013. A análise de conteúdo das respostas destacou: a compreensão dos participantes sobre promoção da saúde aos idosos; a estrutura de trabalho; as atividades desenvolvidas relacionadas à educação em saúde e interdisciplinaridade; a eficácia das práticas que resultou em melhoria da qualidade de vida dos idosos e os desafios para promover as atividades. Apontaram as doenças crônicas, déficit de recursos materiais e humanos. Considera-se que, para promover saúde, é necessário conhecimento, trabalho em equipe e proatividade, para que os resultados possam emergir na qualidade de vida dos idosos.

Palavras-Chave: Idoso; promoção da saúde; interdisciplinaridade; atenção à saúde.
\end{abstract}

ABSTRACT: This quanti-qualitative, descriptive study aimed to ascertain the health promotion practices applied to a group of older adults by an interdisciplinary care team. Twelve 12 health professionals of the senior care group, Amigos de Irmã Fernanda, in Cajazeiras, Paraíba State, answered a questionnaire in 2013. Content analysis of their responses highlighted: the participants' understanding of health promotion for the elderly; the structure of the work; the activities relating to health education and interdisciplinarity; the efficacy of the practices that resulted in improved quality of life for the older adults; and the challenges involved in promoting the activities were chronic diseases, and a lack of human and material resources. It is considered that to promote health requires knowledge, teamwork and proactivity in order for the results to emerge in the older adults' quality of life. Keywords: Older adults; health promotion; interdisciplinarity; health care.

RESUMEN: El objetivo fue conocer las prácticas de promoción de la salud desarrolladas por el equipo interdisciplinario de un grupo de personas mayores. Investigación de tipo cuantitativo/cualitativo, descriptivo, realizada junto a 12 profesionales de la salud del grupo de personas mayores Amigos de Irmã Fernanda de Cajazeiras - Paraíba, quienes respondieron un cuestionario en 2013. El análisis del contenido de las respuestas resaltó: la comprensión de los participantes sobre la promoción de la salud para los ancianos; la estructura del trabajo; las actividades desarrolladas relacionadas con la educación en salud e interdisciplinariedad; la efectividad de las prácticas que resultó en la mejora de la calidad de vida de las personas mayores y los retos para promover las actividades. También apuntaron hacia enfermedades crónicas, recursos humanos y materiales. Se considera que, para promover la salud, son necesarios: conocimiento, trabajo en equipo y proactividad, para que los resultados puedan verse en cuanto a la calidad de vida de las personas mayores.

Palabras Clave: Personas mayores; promoción de la salud; interdisciplinariedad; atención de salud.

\section{INTRODUÇÃO}

O número crescente de idosos no mundo e no Brasil instiga a sociedade a pensar como ofertar atenção e cuidados adequados a essa faixa etária da população, tendo em vista que o envelhecer é uma etapa do ciclo de vida com perdas e ganhos e está inserido em um momento histórico, sociocultural, econômico e político¹.
A atenção à saúde do idoso restringia-se, no passado, à cura da doença, com finalidade essencialmente terapêutica, para a qual a conduta adotada era decidida apenas pelo médico. Nos dias atuais, tendo em vista as múltiplas facetas do processo de envelhecimento, a frequência com que essas situações se

'Enfermeira. Doutoranda do Programa de Pós-Graduação em Enfermagem da Universidade Federal da Paraíba. Docente do Curso de Graduação em Enfermagem da Universidade Federal de Campina Grande. Cajazeiras, Paraíba, Brasil. E-mail: milenascosta2011@hotmail.com.

IIEnfermeira. Doutoranda do Programa de Pós-Graduação em Enfermagem da Universidade Federal da Paraíba. Servidora Técnico-Administrativa da Universidade Federal de Campina Grande. Cajazeiras, Paraíba, Brasil. E-mail: elianeleitesousa@yahoo.com.br.

IIIFisioterapeuta. Docente do Curso de Fisioterapia e Coordenadora do Curso de Pós-Graduação em Fisioterapia Cardiorrespiratória e Hospitalar da Universidade Cruzeiro do Sul. Doutorado em Ciências pela Faculdade de Medicina de São Paulo. Brasil. E-mail: jamilianbar@yahoo.com.

IV Enfermeira. Mestranda do Programa de Pós-Graduação em Enfermagem da Universidade Federal da Paraíba. Docente da Escola Técnica de Saúde da Universidade Federal de Campina Grande. Cajazeiras, Paraíba, Brasil. E-mail: lucosta.ufcg@gmail.com.

vFisioterapeuta. Mestranda do Programa de Pós-Graduação em Enfermagem da Universidade Federal da Paraíba. Docente da Faculdade de Tecnologia da Paraíba. João Pessoa, Paraíba, Brasil. E-mail: ana-margareth@hotmail.com.

${ }^{V I}$ Fisioterapeuta. Doutora em Ciências da Saúde. Docente do Programa de Pós-Graduação em Enfermagem da Universidade Federal da Paraíba, João Pessoa, Paraíba, Brasil.E-mail: jpadelaide@hotmail.com. 
apresentam e suas repercussões sobre o estado de saúde, essa assistência curativa não atende mais às necessidades de saúde do idoso².

A promoção da saúde vem sendo entendida, nas últimas décadas, como uma estratégia promissora para enfrentar os múltiplos problemas de saúde que afetam as populações humanas e seus estornos, em particular, da população idosa ${ }^{3}$. Apresenta como interface a educação em saúde, objetivando a melhoria da autoestima, a redução da alienação e incremento dos conhecimentos, expandindo o campo de possibilidades de escolhas do indivíduo, deixando-o livre para optar sobre seus comportamentos ${ }^{4}$.

Sob um enfoque ampliado, a promoção da saúde é compreendida como uma proposta de educação permanente da comunidade, visando à melhoria de suas condições de vida e saúde. As ações de promoção resultam da combinação de atuações do Estado nas respectivas políticas públicas de saúde; intervenções intersetoriais, atividades comunitárias e dos próprios indivíduos para o desenvolvimento das suas habilidades ${ }^{5}$.

O método considerado mais promissor para realizar a promoção da saúde é a ação educativa, que consiste em atividades dirigidas, centralmente, à transformação dos comportamentos dos indivíduos, focando nos seus estilos de vida. Estas atividades devem ser realizadas em grupo, de forma que suas práticas levem à reflexão sobre o envelhecimento, em suas múltiplas determinações, e estimulem o investimento desejante e participativo na vida ${ }^{3,4}$.

As temáticas abordadas nas ações de saúde do idoso devem abrir espaço ao diálogo efetivo e ultrapassar os assuntos sobre doenças e fatores de risco. São eixos temáticos, tais como envelhecimento, sexualidade, lazer, relações familiares, direitos sociais, assim como inúmeros outros que expressem necessidades e interesse da população idosa.

Nesse prisma, as práticas de promoção da saúde, desenvolvidas por equipes interdisciplinares, passaram a se apresentar como um modelo de atenção ao idoso direcionado para a integralidade, por ter como principal objetivo a manutenção de um bom estado de saúde que, mediante diversas óticas profissionais, utilizam a educação em saúde, visando ao alcance máximo de vida ativa, permitindo-lhe autonomia, independência física, psíquica e social no ambiente em que estiver inserido com sua família ${ }^{5,6}$.

Percebe-se do exposto que, para se oferecer atenção integral à população idosa, a atuação deixa de ser exercida por um ou poucos profissionais e passa a ser executada por uma equipe, cujos componentes serão responsáveis pelas diversas áreas do conhecimento gerontológico, evidentemente trabalhado sob a ótica da interdisciplinaridade.

Frente a estes aspectos, este estudo teve como objetivo conhecer as práticas de promoção da saúde desenvolvidas pela equipe interdisciplinar com um grupo de idosos.

\section{REvisÃo DE Literatura}

O estudo do envelhecimento humano tem se tornado tema pertinente à sociedade atual, dada a elevação da expectativa de vida e tendência ao aumento da população da terceira idade. Entretanto, tal assunto está relacionado à necessidade da formulação, ampliação e fornecimento de políticas públicas de apoio ao idoso, em virtude deles demandarem cuidados específicos ${ }^{1}$.

Nesse contexto, políticas públicas foram criadas para contemplar a saúde do idoso, como, por exemplo, o Pacto pela Defesa da Vida, a Política Nacional de Saúde da Pessoa Idosa e o Estatuto do Idoso. Em todas elas, a promoção da saúde encontra-se como um dos enfoques principais ${ }^{4}$.

A Política Nacional de Promoção da Saúde prevê que, para o indivíduo ter qualidade de vida, incluindo o idoso, é necessário que essa pessoa tenha uma alimentação saudável, prática corporal/atividades físicas, prevenção e controle do tabagismo, redução da morbimortalidade em decorrência do uso abusivo de álcool e outras drogas, por acidentes de trânsito, prevenção da violência e estímulo à cultura da paz, promoção do desenvolvimento sustentável ${ }^{3}$.

\section{Metodologia}

Trata-se de um estudo quantiqualitativo de natureza descritiva, pois as investigações científicas de cunho quantitativo caracterizam-se pela utilização da estatística, que traduz em números as opiniões e informações. Já os estudos de cunho qualitativo buscam compreender e explicar as dinâmicas das relações sociais que, por sua vez, são geradas a partir das ações, crenças, valores, convicções, atitudes e hábitos humanos?

Caracteriza-se como uma pesquisa de campo desenvolvida no grupo de idosos Amigos de Irmã Fermanda, localizado em Cajazeiras - PB. Participaram do estudo 12 profissionais da área da saúde, sendo dois enfermeiros, três educadores físicos, dois fisioterapeutas, dois nutricionistas, um psicólogo, um cirurgião-dentista, um médico. Estes profissionais de saúde compõem o quadro total de voluntários integrantes da equipe interdisciplinar do referido grupo e são oriundos de instituições de ensino superior, como a Universidade Federal de Campina Grande (UFCG) e a Faculdade Santa Maria (FSM); e, ainda, da Secretaria de Educação do Município de Cajazeiras-PB (SEMC). Portanto, não foi necessário adotar procedimentos estatísticos para selecionar os sujeitos do estudo.

As informações foram obtidas por meio de um questionário autoaplicado no mês de janeiro de 2013, após as atividades desenvolvidas no ano de 2012. O instrumento foi construído a partir do objetivo proposto nesta investigação e contemplou questões sobre o perfil sociodemográfico e profissional dos participantes, compreensão, atividades desenvolvidas, eficácia 
das práticas e os desafios para implementar ações de promoção da saúde à pessoa idosa. A cada participante, foi disponibilizado um prazo de 15 dias para a devolução do questionário respondido.

Os dados quantitativos foram analisados de acordo com a estatística descritiva simples em número absoluto e percentual, organizados e apresentados em tabela.

Quanto aos dados qualitativos, utilizou-se a técnica de análise de conteúdo de forma temática, seguindo as etapas de pré-análise, que consistiu na escolha dos documentos a serem analisados conforme o objetivo inicial da pesquisa, ou seja, nessa etapa, foi efetuada a leitura exaustiva das respostas dos questionários dos sujeitos do estudo, procurando-se responder à proposição inicial. Foi feita a exploração do material, que possibilitou a classificação dos dados com vistas a alcançar o núcleo de compreensão do texto. A terceira etapa compreendeu o tratamento dos resultados obtidos, que se pautou na análise dos agrupamentos dos dados contidos nos questionários, estabelecendo relações entre os resultados e a literatura. E, por fim, procedeu-se à interpretação, abordando a análise propriamente dita do estudo ${ }^{8}$. Após compilar os dados, os resultados foram analisados a partir da literatura pertinente.

A análise do conteúdo dos sujeitos fez emergir a elaboração de quatro categorias temáticas, intituladas: Compreensão da promoção da saúde, Atividades de promoção da saúde, Eficácia das práticas interdisciplinares, Dificuldades para as ações de saúde.

A participação dos sujeitos iniciou-se mediante a assinatura do Termo de Consentimento Livre e Esclarecido (TCLE), obtendo-se o parecer favorável $\mathrm{n}^{\circ}$ 0289.0.133.000-11 junto ao Comitê de Ética em Pesquisa da Universidade Estadual da Paraíba/UEPB, conforme doravante preconiza a Resolução n ${ }^{\circ}$ 466/12 do Conselho Nacional de Saúde/Sistema Nacional de Ética em Pesquisa (CNS/SISNEP) que regulamenta a pesquisa com seres humanos no Brasil?

Foi assegurado o anonimato dos profissionais de saúde que participaram do estudo. Os recortes de suas respostas foram identificados pela letra $S$ da palavra sujeito, seguida do número de sequência de devolução do questionário.

\section{Resultados E Discussão}

\section{Caracterização dos profissionais participantes do estudo}

No que se refere à idade dos participantes, predominaram profissionais de saúde adultos jovens, ou seja, de 25 a 35 anos, conforme os dados da Tabela 1.

Considera-se que, na formação acadêmica de décadas anteriores, não havia o incentivo às atividades de promoção da saúde, diferenciando-se da proposta das novas diretrizes curriculares dos cursos da área de saúde ${ }^{10}$. Acrescenta-se que os novos profissionais de saúde também estão sendo preparados para adotar posturas que proporcionem empoderamento aos idosos na promoção de sua saúde ${ }^{5}$. Nesse contexto, foi esperada a predominância do grupo etário declarado por tais participantes.

Quanto ao sexo, 10(83,3\%) participantes eram mulheres, de acordo com a Tabela 1 . Este resultado é esperado quando se trata de profissionais de saúde, pois, apesar de ter aumentado a procura dos homens por tais profissões, ainda há predominância feminina na área de saúde ${ }^{11}$.

Sobre a titulação destes profissionais, $8(66,7 \%)$ eram especialistas em suas áreas e 4(33,3\%) possuíam mestrado em áreas diversas. Estes dados revelam que tais profissionais buscaram a qualificação, para a assistência à população e, em especial, à promoção da saúde do idoso.

A formação de profissionais em gerontologia deve ser uma das prioridades para a evolução das políticas públicas brasileiras de saúde do idoso ${ }^{12}$.

O pensar e o fazer saúde requerem uma nova lógica e organização de trabalho, demandam o desenvolvimento de um processo educacional que possibilite aos profissionais de saúde o aprendizado de outros conhecimentos, saberes e formas de atuação ${ }^{11}$.

Quanto ao tempo de atuação no grupo de idosos, 6(50\%) profissionais realizavam atividades de promoção da saúde entre um e dois anos. Esse dado mostra-se importante, pois expressa sua relevância para a prática do cuidar do idoso, uma vez que as experiências do trabalho viabilizam que o profissional adquira conhecimentos e habilidades para desenvolver suas funções, além de fortalecer o vínculo com essa população, através do estabelecimento de uma relação de confiança, adquirida e aprimorada com o tempo.

TABELA 1: Perfil sociodemográfico e profissional dos participantes que compuseram a equipe interdisciplinar. Cajazeiras - PB, 2012. $(\mathrm{N}=12)$

\begin{tabular}{lcc}
\hline $\begin{array}{l}\text { Variáveis } \\
\text { Idade }\end{array}$ & $\mathbf{f}$ & $\%$ \\
\hline 25 a 35 anos & 6 & 50,0 \\
35 a 45 anos & 3 & 25,0 \\
45 a 55 anos & 2 & 16,7 \\
55 a 65 anos & 1 & 8,3 \\
Sexo & & \\
Masculino & 2 & 16,7 \\
Feminino & 10 & 83,3 \\
Titulação & & \\
Especialização & 8 & 66,7 \\
Mestrado & 4 & 33,3 \\
Tempo atuação no grupo & & \\
> 1 ano & 2 & 16,7 \\
1 a 2 anos & 6 & 50,0 \\
2 a 4 anos & 1 & 8,3 \\
4 a 5 anos & 3 & 25,0 \\
\hline
\end{tabular}


A seguir, foram analisadas e discutidas as quatro categorias temáticas emergentes do estudo, já citadas.

\section{Compreensão da promoção da saúde}

A compreensão dos participantes da pesquisa sobre a promoção da saúde aponta as diversas facetas que contemplam esse tema, pois eles inferiram a uma nova filosofia de trabalho, como um pré-requisito de organização de trabalho e como participação comunitária.

Sabe-se que as pessoas, nos dias atuais, precisam cuidar-se para ter uma velhice com menos problemas, ou seja, sem doenças crônicas. Então, a promoção da saúde é uma forma de se trabalhar com a população, ensinando a eles a se cuidarem. (S1)

A promoção da saúde são ações de saúde sistematizadas e repassadas por um profissional de saúde à população, com objetivo de prevenir doenças e prolongar a vida das pessoas. (S4)

É um tipo de metodologia de trabalho muito eficiente para ensinar e conscientizar as pessoas idosas à prevenção das doenças crônicas degenerativas e deve ser aplicada na atenção primária à saúde por profissionais qualificados. (S7)

Promoção da saúde se faz com ajuda da comunidade. [...] para trabalhar a promoção da saúde, é necessário que todos que atuam em uma equipe de saúde se unam com a comunidade [...]. (S8)

A expressão promoção da saúde começou a ser difundida por Henry Sigerist a partir da década de 1940, porém destacou-se mediante as conferências nacionais e internacionais nas décadas subsequentes e passou a ser inserida na atuação dos profissionais depois do incentivo e compreensão de sua importância para a saúde da população ${ }^{13}$. Apesar do período do surgimento, para alguns dos participantes desta pesquisa, esse tema era novo no cenário de suas atividades ocupacionais.

Estudo realizado reforça o conceito desses profissionais ao dizerem que a concepção da promoção da saúde atual está vinculada a uma combinação de estratégias que envolvem ações do Estado, indivíduo, comunidade, sistema de saúde e parcerias interinstitucionais, com o objetivo de criar ações que possibilitem saúde e, consequentemente, melhor qualidade de vida ${ }^{14}$. No entanto, a participação comunitária é essencial nesse processo, para que as pessoas passem a ter autonomia de propor e decidir as práticas de saúde que mais as beneficiam.

A promoção à saúde objetiva proporcionar a constituição de uma sociedade saudável, na qual todos os cidadãos têm acesso igualitário aos recursos que constituem a qualidade de vida, como educação, habitação e meio ambiente adequado, emprego e renda, informação, lazer, cultura, saneamento, alimentação, segurança, participação social e serviços de saúde, portanto, envolvendo políticas públicas integradas e intersetoriais que atuam nos determinantes sociais da saúde, com ampla participação da comunidade ${ }^{15,16}$.

A promoção da saúde pode também ser vista como outro eixo, ou seja, surge como uma atividade dirigida à mudança de comportamento dos indivíduos, dando ênfase aos seus estilos de vida conforme educação e cultura recebida ${ }^{17}$.

\section{Atividades de promoção da saúde}

As ações implementadas na atenção à saúde do idoso pelos profissionais envolvidos enfocavam a educação em saúde como atividade em comum de todos os profissionais, por eles serem correponsáveis no cuidado ao idoso e ações específicas dos profissionais, como por exemplo, as atividades físicas desenvolvidas pelos educadores físicos, a prevenção de quedas pelos fisioterapeutas, orientações alimentares pelos nutricionistas e consultas realizadas pelos enfermeiros.

Realizamos várias atividades físicas, a exemplo, a caminhada simples e com obstáculo, alongamento, musculação [...] a dança é sempre trabalhado no grupo [...] Através da fisioterapia trabalhamos a prevenção de quedas no idoso. (S9)

As principais atividades que realizo com eles são sobre a prevenção de quedas, posturas. Elas são desenvolvidas no grupo e orientadas para que eles realizem em casa para um melhor desempenho [...]. (S5) Trabalho com ele mostrando o benefício de se ter uma alimentação saudável, então falo do valor dos alimentos, como eles devem ser consumidos, [...]. (S8)

Realizamos a consulta de enfermagem gerontológica, onde fazemos toda a avaliação do idoso. [... . Os idosos são também informados sobre como tomar seus medicamentos, são orientados para cuidar da alimentação e do corpo em geral [...]. (S11)

O conjunto dessas ações possibilita o envolvimento das pessoas idosas no processo da promoção da saúde, bem como a permanência nas ações, que promoverão benefícios e qualidade de vida, sendo, assim, importantes no processo de envelhecimento.

Existem fatores que auxiliam tanto na inserção quanto na adesão do idoso em programas de ações voltados à promoção da saúde, como por exemplo, os aspectos de educação, âmbito familiar, social, saúde, economia. É nessa perspectiva que educar para saúde não é somente informar ou transmitir conhecimentos, mas preparar o indivíduo para o autocuidado ${ }^{18}$.

Um estudo semelhante, realizado em Campinas SP, evidenciou que os profissionais de saúde atuantes na atenção primária de idosos estavam realizando consultas agendadas e eventuais, acolhimento, procedimentos em geral, grupos educativos, atendimento domiciliar, vacinação, avaliação para câncer bucal e ginecológico, 
discussão dos projetos terapêuticos, planejamento e gestão de cuidado ao idoso ${ }^{19}$.

Pesquisa realizada em Portugal revelou que a utilização de cuidados de saúde pelo idoso depende da conjugação de diversos fatores relacionados, como, por exemplo, seu estado de saúde e a disponibilidade da oferta de cuidados de saúde ${ }^{20}$.

\section{Eficácia das práticas interdisciplinares}

As práticas de promoção da saúde realizadas com os idosos pela equipe interdisciplinar melhoraram o desempenho de diversos aspectos, tais como a funcionalidade, a autonomia, independência, bem-estar psicossocial e qualidade de vida dos idosos, pois eles passaram a adotar hábitos saudáveis e mudaram seus estilos de vida.

[...] através destas práticas de saúde, o idoso pode vim a ter vários benefícios sobre sua saúde a exemplo da melhoria na locomoção, melhor desempenho das atividades básicas e instrumentais de vida diária. (S7)

As práticas de saúde apresentam um melhor desenvolvimento de autonomia e independência. (S5)

Ao analisar estes depoimentos, percebeu-se que a realização das práticas em saúde contribuiu para a promoção da saúde, que constitui um dos pilares para um envelhecer saudável e para a qualidade de vida da pessoa idosa.

De acordo com a Política Nacional do Idoso, o envelhecimento bem-sucedido pode ser caracterizado pela presença de boa capacidade funcional física e cognitiva, baixa probabilidade de acometimento de doenças e de incapacidades associadas a elas, autonomia e independência ${ }^{21}$.

Portanto, para que o idoso se mantenha ativo e com uma boa capacidade para desenvolvimento das atividades instrumentais e básicas de vida diária, é necessário que ele esteja envolvido em programas que contemplem a interdisciplinaridade.

O envelhecimento com qualidade de vida é uma aspiração imprescindível, que potencializa o viver, e depende, em grande parte, de condições sociais e políticas públicas que garantam direitos básicos de cidadania e possibilitem práticas tendencialmente saudáveis, como alimentação equilibrada, atividade física, uso prazeroso do corpo, inserção social e ocupacional dotada de serviços assistenciais e preventivos ${ }^{13,22}$.

A promoção da saúde emerge de forma primordial para prevenir e controlar os problemas de saúde comuns nessa etapa da vida. Nesse sentido, promover o envelhecimento saudável é tarefa complexa, que inclui a conquista de uma boa qualidade de vida e o amplo acesso aos serviços, que favoreçam lidar com as questões do envelhecimento da melhor maneira possível ${ }^{3}$. Essas considerações colocam, diante do desafio de vivenciar o envelhecimento, a importância das ações de promoção de saúde, para manutenção da saúde do idoso.

\section{Dificuldades para as ações de saúde}

As dificuldades enfrentadas na realização das ações de saúde pelos participantes estavam relacionadas aos problemas de saúde vivenciados pelos idosos, déficit de recursos materiais e físicos.

Um dos entraves que enfrentamos no grupo é o pequeno espaço físico para o desenvolvimento das ações de saúde pelos profissionais. (S3)

A pessoa na terceira idade tem muitas doenças crônicas que atrapalham nas atividades físicas que são realizadas no grupo [...]. (S4)

As maiores dificuldades que eu vejo, está nas limitações dos idosos, a exemplo de pouca visão, audição, locomoção. Tudo isso interferem nas atividades desenvolvidas no grupo. (S11)

Temos enfrentado algumas dificuldades no que diz respeito à falta de alguns equipamentos que são necessários para realizar determinadas atividades tanto físicas como de fisioterapia [...]. (S12)

As enfermidades crônicas em idosos apresentam-se como um sério problema de saúde, que necessita ser diagnosticado, mensurado, avaliado e tratado pelos profissionais, preferencialmente por uma equipe interdisciplinar, uma vez que são esses os agentes capazes de, através de intervenções, minimizar a morbidade e melhorar a qualidade de vida desses indivíduos.

Um estudo epidemiológico revelou que as doenças que mais acometem a população idosa estão associadas às desordens crônicas, particularmente doenças musculoesqueléticas, como artrites, artrose, osteoporose, diabetes e hipertensão arterial $^{23}$.

As doenças crônicas, quando presentes na vida do idoso, instigam, consomem, enfraquecem, fragilizam e ameaçam sua segurança, autonomia e independência, impedindo-o, muitas vezes, de realizar atividades instrumentais e de vida diária, bem como limitando a capacidade de interação e convívio social, diminuindo, consideravelmente, sua qualidade de vida ${ }^{24}$.

No entanto, o envelhecer não pode ser visto como doença, mas como uma etapa da vida com características e valores próprios, em que ocorrem modificações nas condições orgânicas e emocionais do indivíduo ${ }^{25}$.

Os participantes citaram, também, como dificuldade, o déficit de condições de trabalho, o que pode impedir o desempenho satisfatório nas ações de saúde prestada por partes desses profissionais.

Em um estudo, desenvolvido em Campinas - SP, sobre a atuação das equipes de saúde da família junto aos idosos, encontrou-se como principal dificuldade para implementação das ações de promoção da saúde o número limitado de recursos humanos e, em segundo lugar, a infraestrutura e equipamentos, assemelhando-se ao presente estudo ${ }^{19}$. 
Na Colômbia, a realidade não difere, em grande escala, da do Brasil, pois apesar do reconhecimento e sucesso em várias regiões dos programas de atenção primária em saúde, as dificuldades são evidentes e semelhantes às brasileiras ${ }^{26}$.

Os problemas citados pelos participantes e demais autores podem estar relacionados à crise econômica em que os países vivem e repercutem sobre a saúde da população. A redução dos serviços coloca tensão na capacidade dos atores individuais e coletivos responderem às dificuldades, em que se incluem, com particular expressão, as de acesso aos serviços de saúde ${ }^{27}$.

\section{Conclusão}

A compreensão dos participantes sobre promoção da saúde aos idosos enfocou: estrutura de trabalho; as atividades desenvolvidas relacionadas à educação em saúde e ações interdisciplinares; a eficácia das práticas para a melhoria da qualidade de vida dos idosos e os desafios enfrentados para a promoção da saúde. Esses temas consideraram as especificidades das doenças crônicas e a carência de recursos materiais e humanos.

Como limitações do estudo, evidenciaram-se lacunas no tocante às propostas das políticas públicas de saúde voltadas para a pessoa idosa, que podem ter sido decorrentes da falta de investimento dos gestores e/ou por déficit de atuação com esse público.

Na promoção da saúde, ainda há muito por fazer, visto que sua característica multifacetada deflagra entraves que perpassam pelos aspectos sociais, culturais e econômicos. Por outro lado, o exercício da promoção da saúde sob o enfoque multidisciplinar agrega novas possibilidades, na medida em que a educação em saúde e as práticas interventivas específicas de cada profissão conseguem melhorar a funcionalidade, autonomia e independência do idoso, contribuindo para seu bem-estar psicossocial.

Promover saúde requer conhecimento, empenho e capacidade proativa e, quando realizada em equipe interdisciplinar, necessita, ainda, que seus agentes promotores concentrem esforços com vistas à integralidade e à melhoria da qualidade de vida da pessoa idosa.

\section{REFERÊNCIAS}

1.World Health Organization. Organização Pan-Americana da Saúde. Envelhecimento ativo: uma política de saúde. Brasília (DF): WHO; 2005.

2.Papaléo Netto M, Yuaso DR. Interdisciplinaridade em gerontologia: aspectos conceituais e objetivos. In: Papaléo Netto M. Tratado de gerontologia. São Paulo: Editora Atheneu; 2007. p. 149-62.

3.Gutierrez PR, Oberdiek HI. Concepções sobre a saúde e a doença. In: Andrade SM, Soares DA, Cordoni
Júnior L. Bases da Saúde Coletiva. Londrina (PR): Ed. UEL; 2001. p.1-25.

4. Teixeira MB. Empoderamento de idosos em grupos de promoção da saúde [dissertação de mestrado]. Rio de Janeiro: Escola Nacional de Saúde Pública; 2002.

5.Santos SSC, Barlem ELD, Silva BT, Cestari ME, Lunardi VL. Promoção da saúde da pessoa idosa: compromisso da enfermagem gerontogeriátrica. Acta Paul Enferm. [Scielo-Scientific Electronic Library online] 2008 [citado em 18 jan 2015]. 21:649-53. Disponível em: http://www.scielo.br/scielo.php?pid=S0103$-21002008000400018 \&$ script $=$ sci_arttext.

6.Assis M. Envelhecimento ativo e promoção da Saúde: reflexões para ações educativas com idosos. Rev APS. 2005; 8:15-24.

7.Costa SFG, Silva MIT, Oliveira EF, Lima CB. Metodologia da pesquisa: coletânea de termos. João Pessoa (PB): Ideia; 2000.

8.Minayo MCS, Deslandes SF, Gomes R. Pesquisa social: teoria, método e criatividade. Petrópolis (RJ): Vozes; 2007.

9.Ministério da Saúde (Br). Conselho Nacional de Saúde. Comissão Nacional de Ética em Pesquisa. Normas para pesquisa envolvendo seres humanos: Resolução $\mathrm{n}^{\mathrm{O}}$ 466/12 do Conselho Nacional de Saúde. Brasília (DF): Ministério da Saúde; 2012.

10.Mattos D, Aguiar AC. As novas diretrizes curriculares e a integralidade em saúde: uma análise das possíveis contribuições da odontologia para o trabalho em equipe [dissertação de mestrado]. Rio de Janeiro: Universidade do Rio de Janeiro; 2006.

11.Cotta RMM, Schott M, Azeredo CM, Franceschini SCC, Priore SE, Dias G. Organização do trabalho e perfil dos profissionais do Programa Saúde da Família: um desafio na reestruturação da atenção básica em saúde. Epidemiol Serv Saúde. 2006; 15:7-18.

12.Cassab AK. The accelerated aging of the population in Brazil. Soins Gerontol. 2013; 100:32-5.

13.Texeira CF, Solla JP. Modelo de atenção à saúde: promoção, vigilância e saúde da família. Salvador (BA): EDUFBA; 2006.

14.Buss PM. Uma introdução ao conceito de promoção da saúde. In: Czeresnia D, Freitas CM; organizadores. Promoção da saúde: conceitos, reflexões, tendências. Rio de Janeiro : Editora Fiocruz; 2003. p.117-39.

15.Ministério da Saúde (Br). Secretaria de Vigilância em Saúde. Política Nacional de Promoção a Saúde. Brasília (DF): Secretaria de Atenção à Saúde; 2006.

16.Cruz ALB, Martins KL. Percepção da promoção da saúde do idoso: olhar de agentes comunitários de saúde. Rev enferm UFPE. 2010; 4:1484-91.

17.Lemos LA, Fiuza MLT, Pinto ACS, Galvão MTG. Grupo de promoção da saúde para portadores do vírus da imunodeficiência humana. Rev enferm UERJ. 2013; 21:521-26.

18.Michel RS. Portal Fórum [site de Internet]. O desafio das barreiras. [citado em 10 mai 2015]. Disponível em: http:// www.portaldoenvelhecimento.net/pforum/afv2.htm. 
19.Garcia MAM, Miyamoto DA, Frigério RM, Merlin SS. A atuação das equipes de saúde da família juntos aos idosos. Revista APS. 2006; 9:4-14.

20.Quintal C, Lourenço O, Ferreira P. Utilização de cuidados de saúde pela população idosa portuguesa: uma análise por género e classes latentes. Rev port Saude Publica. 2012; 1:35-46.

21. Ministério da Saúde (Br). Portaria de $n^{0} 2.528$ de 19 de outubro de 2006. Aprova a Política Nacional de Saúde da Pessoa Idosa e dá outras providências. Brasília (DF): Gabinete Ministerial; 2006.

22.Moliterno ACM, Faller JW, Borghi AC, Marcon SS, Carreira L. Viver em família e qualidade de vida de idosos da Universidade Aberta da Terceira Idade. Rev enferm UERJ. 2012; 20:179-84.
23.Gold DT, Roberto K A. Correlates and consequences of chronic pain in older adults. Geriatr Nurs. 2005; 21:270-3. 24.Celich KLS, Galon C. Dor crônica em idosos e sua influência nas atividades da vida diária e convivência social. Rev Bras Geriatr Gerontol. 2009; 12:345-60.

25.Oliveira ALB, Dourado MB, Menezes TMO. A percepção dos graduandos de enfermagem sobre envelhecimento. Rev enferm UERJ. 2014; 22:680-5.

26.Campos AC, Soler JMA, Moreno YAC. A look at primary health care and reflections on its development in Colombia. Revista de Actualizaciones en Enfermería. 2013; 16:50-6.

27.Loureiro I, Miranda N, Miguel JMP. Promoção da saúde e desenvolvimento local em Portugal: refletir para agir. Rev port Saude Publica. 2013; 31:23-31. 